\title{
CII, CI, and $\mathrm{CO}$ in the massive star forming region W3 Main
}

\author{
Carsten Kramer $^{1}$, Holger Jakob ${ }^{1}$, Bhaswati Mookerjea ${ }^{1}$, Nicola Schneider ${ }^{2}$, \\ Martin Brüll ${ }^{1}$, and Jürgen Stutzki ${ }^{1}$ \\ 1 Universität zu Köln, I. Physikalisches Institut, Zülpicher Strasse 77, 50937 Köln \\ kramer@ph1.uni-koeln.de, jakob, bhaswati, bruell, stutzki \\ 2 Observatoire de Bordeaux, Universite de Bordeaux 1, BP 89, 33270 Floirac, \\ France schneider@obs.u-bordeaux1.fr
}

\section{Introduction}

We used the KOSMA $3 \mathrm{~m}$ telescope to map the core $7^{\prime} \times 5^{\prime}$ of the Galactic massive star forming region W3 Main in the two fine structure lines of atomic carbon and four mid- $J$ transitions of $\mathrm{CO}$ and ${ }^{13} \mathrm{CO}$. In combination with a map of singly ionized carbon (Howe et al. 1991), and ISO/LWS data at the center position, these data sets allow to study in detail the physical structure of the photon dominated cloud interface regions (PDRs) where the occurance of carbon changes from CiI to Ci to CO. The physical and chemical processes in these layers are governed by FUV radiation. Depending on the induced chemistry, the critical densities, and energy levels of their transitions, the lines of $[\mathrm{CII}],[\mathrm{CI}]$, and $\mathrm{CO}$ serve as probes to trace the different temperature and density regimes.

W3 Main is a well known site of high-mass star formation (e.g. Tieftrunk et al. 1998). It is associated with the W3 giant molecular cloud complex within the Perseus arm at a distance of $2.3 \mathrm{kpc}$ (Georgelin \& Georgelin 1976, cf. Imai et al. 2000). W3 Main comprises several HII regions of different sizes and evolutionary stages, some of them hypercompact, possibly identifying the earliest stages of high-mass star formation (Tieftrunk et al. 1997).

\section{Modelling the spatial structure of the FUV field}

Figure 1 shows the positions of the 12 OB stars found in W3 Main (Tieftrunk et al. 1998). The most luminous source is IRS 2, identified as O5 star. The luminosity of IRS 5 at the map center position corresponds to a cluster of $\sim 7$ B0-type stars (Claussen et al. 1994). We have used the spectral types of the exciting $\mathrm{OB}$ stars to calculate the spatial large-scale variation of the 
far-UV field heating the molecular cloud. Figure 1 shows the resulting FUV distribution relative to that of [CII] emission (Howe et al. 1991).

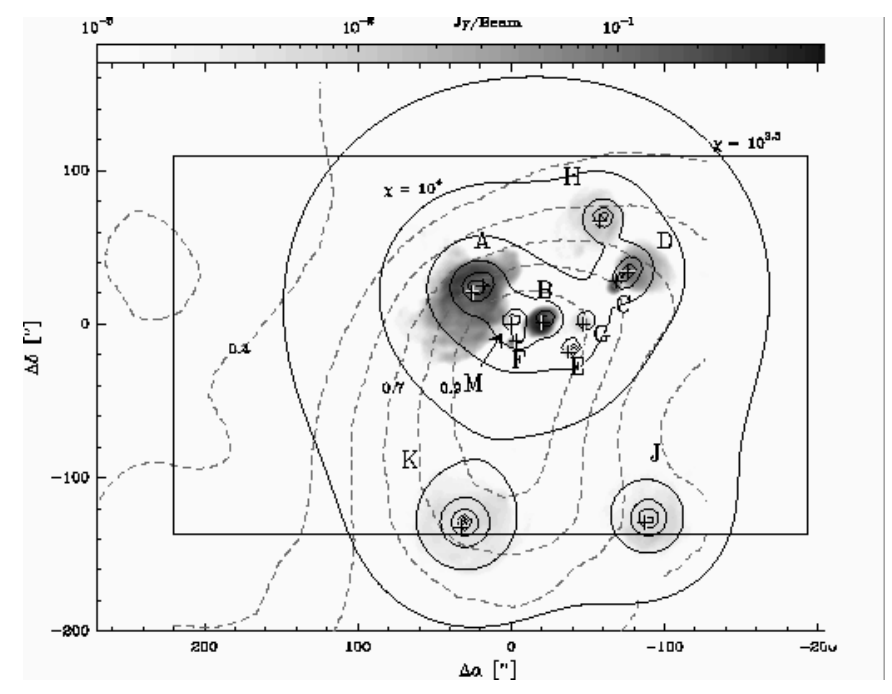

Fig. 1. Overlay of UV field modelled from the spectral types of the OB stars known in W3 Main, [CII] emission, and $6 \mathrm{~cm}$ radio continuum emission. Grey-scale: Image of the VLA $6 \mathrm{~cm}$ radio continuum (Tieftrunk et al. 1997). The box denotes the region mapped with KOSMA in CO $3-2,4-3,7-6$, [CI] $1-0,2-1$, and in ${ }^{13} \mathrm{CO} 8-$ 7. Solid contours: Contours of the modelled spatial structure of the FUV field derived from the spectral types of the embedded OB stars (positions are marked with $\mathrm{a}+$ ). The UV flux $\chi$ varies between $10^{3.5}$ at the cloud edge and $10^{6}$ in the immediate vicinity of IRS 2 . Attenuation by the cloud density structure has yet not been taken into account. Dashed contours: extended [CII] emission observed by Howe et al. (1991) with the KAO at $80^{\prime \prime}$ resolution.

\section{IRS 5 in W3 Main}

\subsection{KOSMA and ISO/LWS data}

Figure 2 shows representative spectra at the center position IRS $5 .{ }^{12} \mathrm{CO}$ 3-2 peak temperatures indicate an excitation temperature of at least $50 \mathrm{~K}$. A lower limit of the $[\mathrm{CI}]$ excitation temperature of $122 \mathrm{~K}$ has been derived from the [CI] 2-1/1-0 line ratio assuming a calibration error of $20 \%$. Column densities per $75^{\prime \prime}$ beam $(0.84 \mathrm{pc})$ and the relative abundances of the three major gas phase species CO, CI, and CII, are derived assuming LTE. At IRS 5, the CII:Cr:CO column density ratio is 18:8:74, i.e. most of the gasphase carbon is in CO, $\sim 20 \%$ is in CII, and only less than $10 \%$ is in atomic 
carbon. The total $\mathrm{H}_{2}$ column density derived from ${ }^{13} \mathrm{CO} 3-2$ observations is $410^{22} \mathrm{~cm}^{-2}\left(\mathrm{~A}_{V} \sim 40 \mathrm{mag}\right)$ within the $75^{\prime \prime}$ beam which agrees well with the result of Tieftrunk et al. (1998) derived from $\mathrm{C}^{18} \mathrm{O}$ observations.

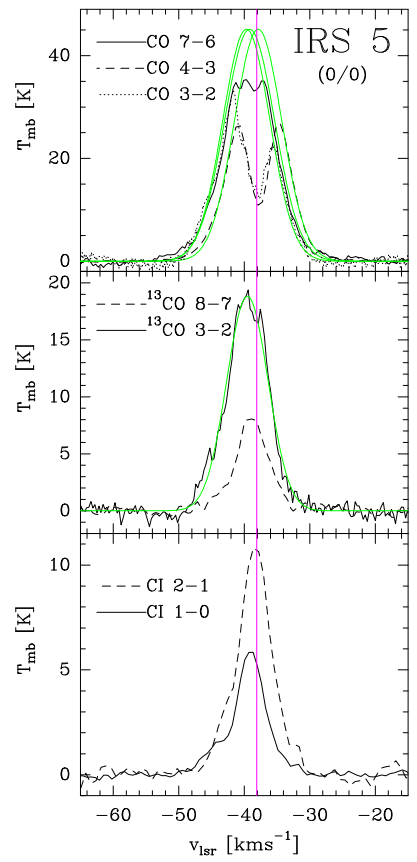

Figure 2: Spectra at one representative position: IRS 5 in W3 Main. All spectra are on a common spatial resolution of $75^{\prime \prime}(0.84 \mathrm{pc})$ and are plotted on the $T_{\mathrm{mb}}$ scale. ${ }^{13} \mathrm{CO}$ and $\mathrm{CI}$ lines peak at the velocity of a dip in the ${ }^{12} \mathrm{CO}$ spectra, indicating that the dip is due to a colder foreground component at about $38 \mathrm{~km} \mathrm{~s}^{-1}$ (denoted by a vertical line). To estimate the line integrated ${ }^{12} \mathrm{CO}$ intensity of the background source, we fitted a Gaussian to the high and low velocity parts of the ${ }^{12} \mathrm{CO}$ spectra. The fit results are shown.

We combined the KOSMA data at IRS 5 with ISO/LWS data from the ISO data archive taken at the same position. Several fine structure lines of [OIII], $[\mathrm{NIII}]$, and $[\mathrm{NII}]$ were detected tracing the ionized medium, as well as lines of $[\mathrm{CII}],[\mathrm{OI}]$, and four high- $J$ rotational transitions of $\mathrm{CO}$ tracing the neutral medium at the PDR cloud surfaces. Figure 3 shows the observed $\mathrm{CO}$ and ${ }^{13} \mathrm{CO}$ fluxes together with the results of a homogeneous, non-LTE radiation transfer model. The detected line strengths indicate kinetic temperatures of more than $140 \mathrm{~K}$ and densities of more than $310^{4} \mathrm{~cm}^{-3}$.

\subsection{Detailed PDR Modelling - First results}

We have started to determine the physical structure of the PDR by detailed modelling using the FUV field previously derived. Here, we briefly describe first results obtained from modelling the emission at IRS 5 using the spherical Cologne PDR model (Störzer et al. 1996, 2000). Its basic free parameters are the clump surface density, its total mass, and the impinging FUV field. We kept the FUV field fixed at $10^{5}$ times the Draine field as derived from the contribution of all embedded OB stars. First results indicate a common solution 


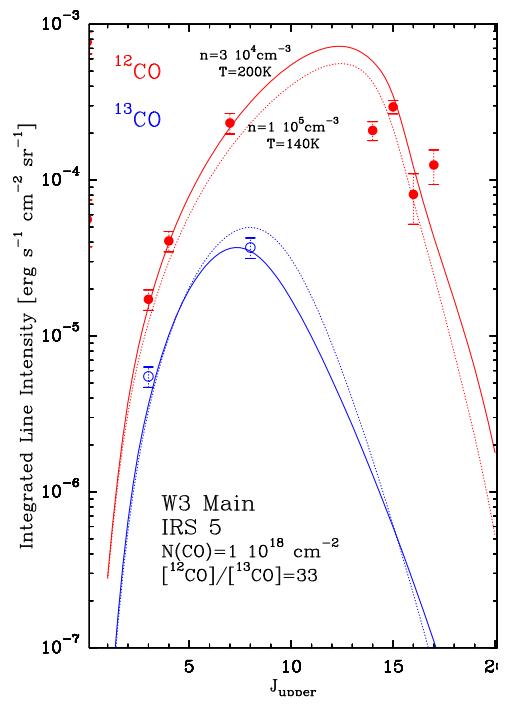

Figure 3: Integrated CO intensities as a function of upper rotational quantum number $J$ for IRS 5 in W3 Main and CO cooling curves produced by a non-LTE radiative transfer model. Rotational transitions upto $J=8$ were observed with KOSMA, the high- $J$ transitions upto $J=17$ were observed with ISO/LWS.

for most ratios (see Figure 4). Future modelling will include the absolute line intensities and explore the possible importance of clumps of different masses (clump mass spectrum), and of pre-shielding by an embedding $\mathrm{H}_{2}$ interclump medium. 


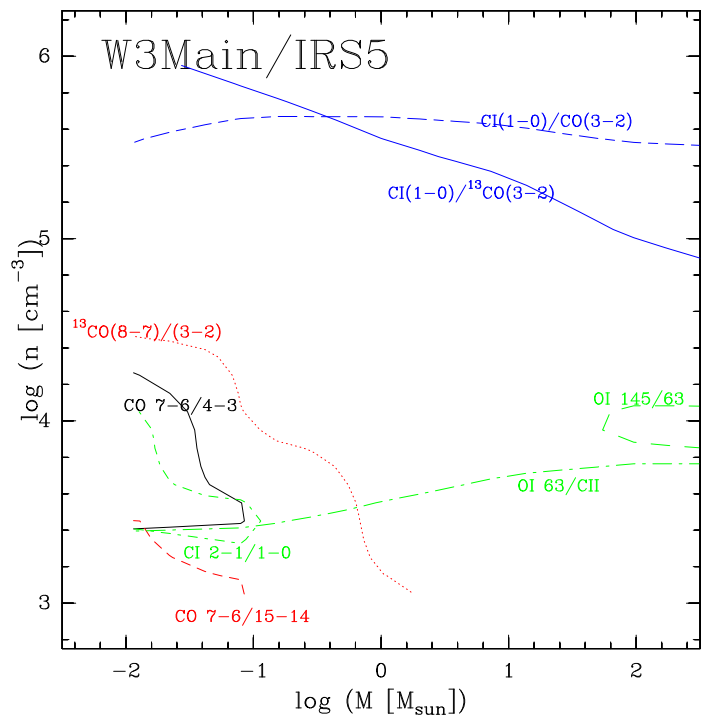

Figure 4: Results from the PDR model for an external FUV field of $\chi=10^{5} \chi_{0}$. Lines denote the observed line ratios at IRS 5 as a function of clump mass and clump surface density. The $[\mathrm{CI}] / \mathrm{CO}$ and $[\mathrm{CI}] /{ }^{13} \mathrm{CO}$ flux ratios indicate high volume densities of more than $10^{6} \mathrm{~cm}^{-3}$. The [OI $145 \mu \mathrm{m} /$ OI $63 \mu \mathrm{m}$ ] ratio is larger than expected, probably due to optically thick [OI] $63 \mu \mathrm{m}$ emission and self-absorption due to the colder foreground material also responsible for the dips in the low- and mid- $J$ CO lines. All other five observed ratios indicate a common solution of about $n \approx 10^{4} \mathrm{~cm}^{-3}$ and a clump mass of less than $\approx 0.1 \mathrm{M}_{\text {sun }}$ in this preliminary analysis. 